Research Article

\title{
Feedback Control of Injection Rate of the Injection Molding Machine Based on PID Improved by Unsaturated Integral
}

\author{
An Yang $\mathbb{D},{ }^{1}$ Weigang Guo, ${ }^{1}$ Tianlong Han, ${ }^{1}$ Congrong Zhao, ${ }^{1}$ Hongwei Zhou, ${ }^{2}$ \\ and Jianping $\mathrm{Cai}^{3}$ \\ ${ }^{1}$ Hangzhou Vocational and Technical College, Hangzhou 310018, China \\ ${ }^{2}$ Tederic Machinery Co., Ltd., Hangzhou 310018, China \\ ${ }^{3}$ Ningbo Haitian Plastic Machinery Group Co., Ltd., Ningbo 315821, China
}

Correspondence should be addressed to An Yang; 2007010028@hzvtc.edu.cn

Received 17 March 2021; Accepted 12 May 2021; Published 25 May 2021

Academic Editor: Ling Zhou; lingzhou@ujs.edu.cn

Copyright (c) 2021 An Yang et al. This is an open access article distributed under the Creative Commons Attribution License, which permits unrestricted use, distribution, and reproduction in any medium, provided the original work is properly cited.

In conventional proportional-integral-derivative (PID) control, the integral term occupies a significant amount of controller memory, which prolongs the calculation time. The integral term easily leads to overshooting and oscillation, while the derivative term reduces the controller's anti-interference ability. In view of these problems, a PID expression with a recurrence relationship was derived, and the integral and differential terms of the conventional PID model were improved using an unsaturated integral and passivation differential to achieve a good control effect. Then, the improved PID was applied to the injection speed control of an injection molding machine, which is usually controlled using conventional PID control that featured difficulty in mathematical modeling, a nonlinear relationship between the input and the output, and high system complexity. Taking an injection molding machine as the control object, the transfer function of the injection system was constructed. Then, the improved PID was simulated using Matlab/Simulink. Lastly, the improved PID was verified using experiments. The simulation and the experimental results showed that the control model had a rapid response, no overshoot, and a high precision.

\section{Introduction}

To make the parameters in the controlled object behave in an ideal way, it is necessary to use corresponding control methods, such as feedforward control [1], proportionalintegral-derivative (PID) control [2,3], robust control [4, 5], adaptive control $[6,7]$, and compound control $[8,9]$. Among the control methods, feedforward control is a type of openloop control. It uses a hysteresis model, which is easy to implement and does not require a sensor. However, it cannot completely eliminate control errors and it is difficult to use to achieve high-precision control. PID control, robust control, and adaptive control are types of feedback control that can theoretically eliminate control errors and meet the requirements of high-precision control. However, the cost of the control system is increased due to its need for highprecision sensor feedback. Compound control is a control method combining feedforward control with feedback control that can obtain good comprehensive performance, although the control algorithm is often complex. PID control technology is advanced, and it does not require an accurate model of a controlled object. Moreover, it features easy parameter tuning, a simple algorithm, and good stability. Therefore, PID control is widely used. In conventional PID control, however, the integral term occupies excessive memory for the controller, which results in a series of problems, such as overshoot and oscillation being easily caused by the integral term, as well as the decrease in the anti-interference ability of the controller caused by the differential term. To solve the problem of unsatisfactory parameter tuning, different forms of improvement have been tried. Han et al. [10] used a search algorithm using particle swarm with $\mathrm{H} 2$ control standards objective function to optimize PID parameters, and the improved PID was used to control the water hydraulic position servo system which is intent to be used in remote control robot for fusion reactor. 
Compared with conventional parameter tuning, the control effect of the PID significantly improved. Ajmeri and Ali [11] established a new cascade control structure for the PID of a first-order integral system. Due to the use of the PID controller in the forward channel, although the response of this control structure was faster, there were problems such as the increase of overshoot and the degradation of the disturbance suppression performance. Nunn and Grolman [12] proposed to control the injection speed by time periods under different injection molding environments and different raw materials, based on the characteristics of the injection speed, However, this control strategy still does not solve the injection accuracy, stability, and the problem of overshoot. In order to control the quality of plastic parts, Kamal et al. [13] proposed a cascade control system design scheme. By constructing the transfer function of the injection system and the transfer function of the temperature system, the injection speed and the body temperature between cycles are controlled.

Based on the research of the above scholars, the control of injection speed has its own advantages and disadvantages. At present, traditional PID control is still the most commonly used control strategy in actual production for injection speed control. Therefore, this paper studies the highorder system model of injection speed, based on the analysis of the shortcomings of traditional PID controllers: slow response speed, large overshoot, poor robustness, and weak anti-interference ability. Through the modification of the integration and differentiation term of traditional PID, the improved PID has the advantages of rapid response, strong anti-interference ability, small overshoot, and good robustness.

\section{The Problem of Injection Speed Control of Injection Molding Machine}

Injection molding of the injection molding machine can be divided into four stages: preheating, injection, packing, and cooling. As shown in Figure 1, in the second stage of injection molding, the screw in the barrel injects the preheated and melted raw materials into the cavity of the mold through the nozzle. During this stage, the mainly controlled parameters are injection speed and injection pressure. After the melted raw material fills the mold cavity, the injection hydraulic cylinder needs to maintain pressure for a period of time to prevent the raw material from flowing back until the raw material is cooled and solidified. The ejector pin then ejects the solidified part from the mold cavity and the process continue to repeat from the first stage. In the injection molding process, the control of the injection speed directly determines the quality of the molded product. The characteristics of the entire injection process are analyzed. The injection speed has the following characteristics of repeated operation, simple and stable, after the analysis of the entire injection process. The shortcomings of the traditional PID control of injection speed are improved and applied to the control of the injection speed.

\section{Improvement of the PID Controller}

3.1. PID of Recurrence Relationship. The PID controller performed proportional adjustment, integral adjustment, and differential adjustment on the difference $e$ between the target value and the actual value, and the adjusted combination was used as the input value $u$ of the system. The equation could be expressed as follows:

$$
u(k)=K_{P}\left\{e(k)+\frac{T}{T_{\mathrm{I}}} \sum_{i=0}^{k} e(i)+\frac{T_{D}}{T}[e(k)-e(k-1)]\right\},
$$

where $u(k)$ is the system input variable of the $k^{\text {th }}$ sampling period, $T$ is the sampling period and its corresponding $k$ denotes sampling sequence number, kT represents the $k^{\text {th }}$ sampling period, $e(k)$ and $e(k-1)$ are the deviations between the target value and the actual value at the $k^{\text {th }}$ and $k-1^{\text {th }}$ sampling, respectively, $K_{P}$ is the proportional coefficient, $T_{I}$ is integral time constant, and $T_{D}$ is the differential time constant.

To obtain the output of the PID control given in (1) at a certain time, it was necessary to integrate all the deviations of the past time and the current time, which not only occupied a significant amount of computer memory but also took a long time to calculate, making it difficult to achieve fast control. For this reason, a PID with a recurrence relationship was adopted in this study. According to (1), the output of the PID controller at time $(k-1) T$ was

$$
u(k-1)=K_{\mathrm{P}}\left\{e(k-1)+\frac{T}{T_{\mathrm{I}}} \sum_{i=0}^{k} e(i)+\frac{T_{D}}{T}[e(k-1)-e(k-2)]\right\} .
$$

Using the difference between (1) and (2), the PID difference could be expressed as

$$
u(k)=u(k-1)+K_{P}\left\{e(k)-e(k-1)+\frac{T}{T_{\mathrm{I}}} e(k)+\frac{T_{D}}{T}(e(k)-2 e(k-1)+e(k-2))\right\} .
$$




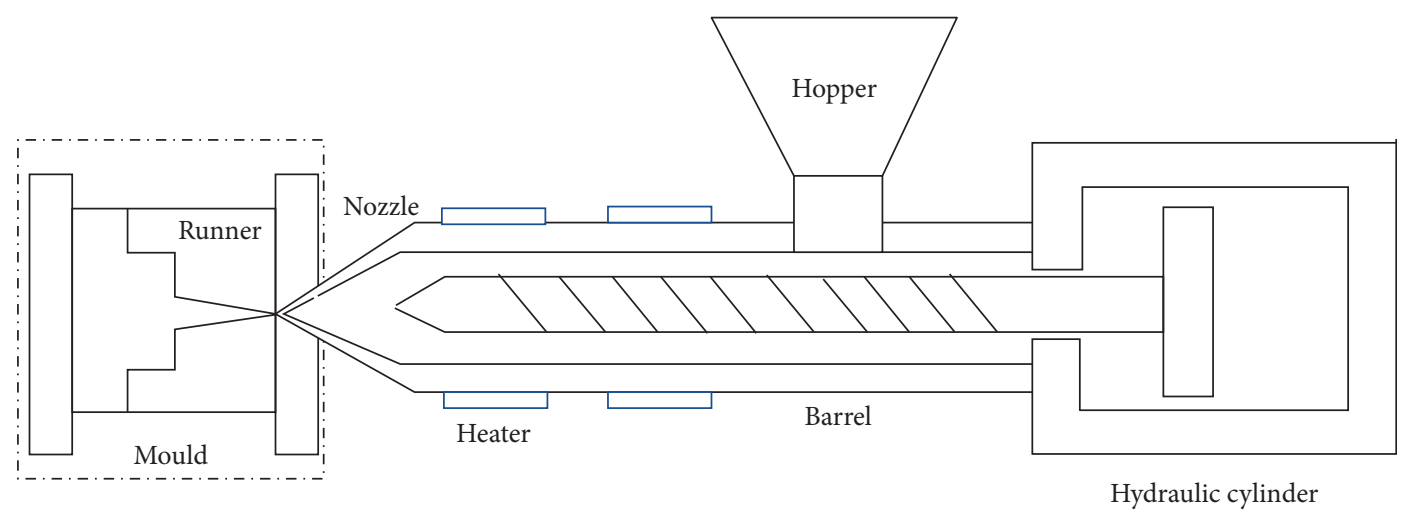

FIGURE 1: Schematic diagram of injection molding machine.

Letting $m_{1}=-K_{P}\left(1+2\left(T_{D} / T\right)\right)$ and $m_{2}=K_{P}\left(T_{D} / T\right)$, then (3) could be expressed as follows:

$$
u(k)=u(k-1)+m_{0} e(k)+m_{1} e(k-1)+m_{2} e(k-2) .
$$

According to (4), there were only four parameters that determined the system input variables $u(k)$, namely, $e(k), e$ $(k-1), e(k-2)$, and $u(k-1)$, which greatly reduced the calculation time of the controller, thus greatly improving the control speed and making fast control easier.

After performing a $\mathrm{Z}$ transformation on (4), the transfer function of the PID could be obtained:

$$
\frac{U(z)}{E(z)}=\frac{m_{0}+m_{1} z^{-1}+m_{2} z^{-2}}{1-z^{-1}} .
$$

3.2. Integral Term Improvement of the PID Controller. The integral term in the PID controller was used to eliminate the steady-state error in the control. However, when the target value changed drastically in a short period of time, a huge fluctuation deviation $e$ is produced, leading to a large overshoot or oscillation of the output value that affect the control effect seriously. Therefore, the integral term was improved to eliminate the steady-state error and control the overshoot.

In order to enable the integral term to achieve improvement described above at the same time, an unsaturated integral was used to improve the conventional integral in the PID controller. Its algorithm is shown in Figure 2. When calculating the control quantity $u(k)$, the first step was to determine whether the control quantity $u(k-1)$ was greater than its maximum set value $u_{\max }$. If $u(k-1) u_{\max }$, only the positive deviation was integrated; if $u(k-1)>u_{\max }$, only the negative deviation was integrated.

3.3. Differential Term Improvement of the PID Controller. Although the differential term in the PID controller could accelerate the response speed of the system, it would lower the controller's anti-interference ability. In the control process, the external environment was inevitably interfered with. Excessive external interference could cause significant changes in the value of the input variable $u$ and rapid changes in the frequency. It had a great impact on the control accuracy of the system, especially for the actual production system. It would not only damage the production system but also harm the personal safety of production personnel. The conventional differential term was improved based on its shortcomings.

In the actual control process, the external interference signals were mostly high-frequency signals, and the low-pass filter was an effective means for removing high-frequency signals. Therefore, in order to eliminate the adverse effect of the disturbance on the differential link, the passivation differential was used to improve the conventional differential in the PID controller, as shown in Figure 3. The lowpass filter was introduced into the conventional PID controller to filter out the interference signals so that the differential term could improve the dynamic characteristics of the system while reducing its sensitivity to interference.

By discretizing the low-pass filter in Figure 3 and substituting $S=(1-z-1) / T$ into the low-pass filter equation, the following equation could be obtained:

$$
\frac{U(z)}{U^{1}(z)}=\frac{1}{\tau^{1}(1 / T)\left(1-z^{-1}\right)+1}=\frac{1}{\left(1+\left(\tau^{1} / T\right)\right)-\left(\tau^{1} / T\right) z^{-1}}=\frac{T}{T+\tau^{1}} \frac{T}{1-\alpha z^{-1}},
$$

where $\alpha=\left(\tau^{1} / T\right)\left(1 / 1+\tau^{1} / T\right)$. 


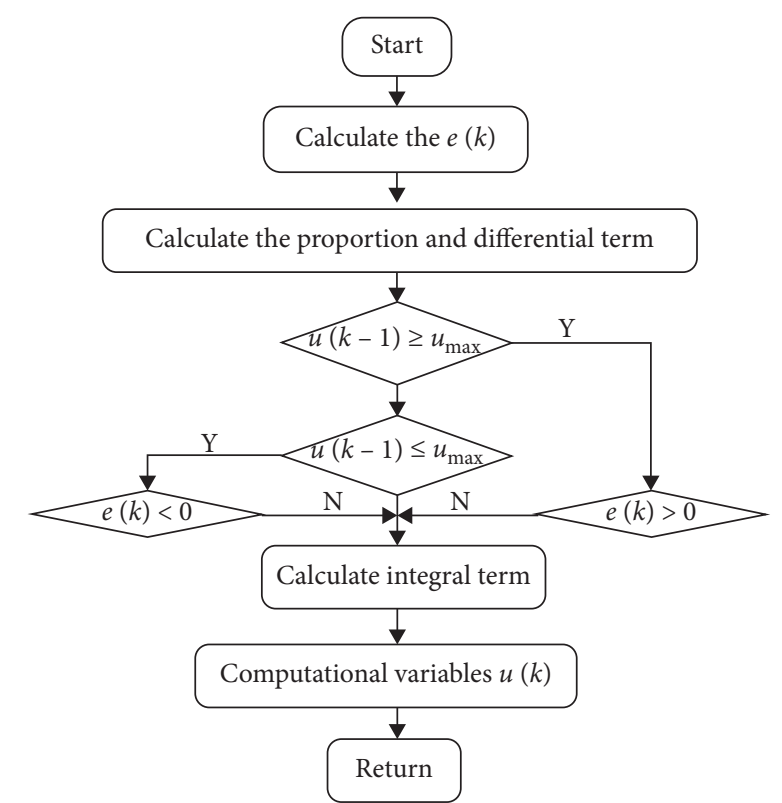

FIGURE 2: Block diagram of unsaturated integration algorithm.

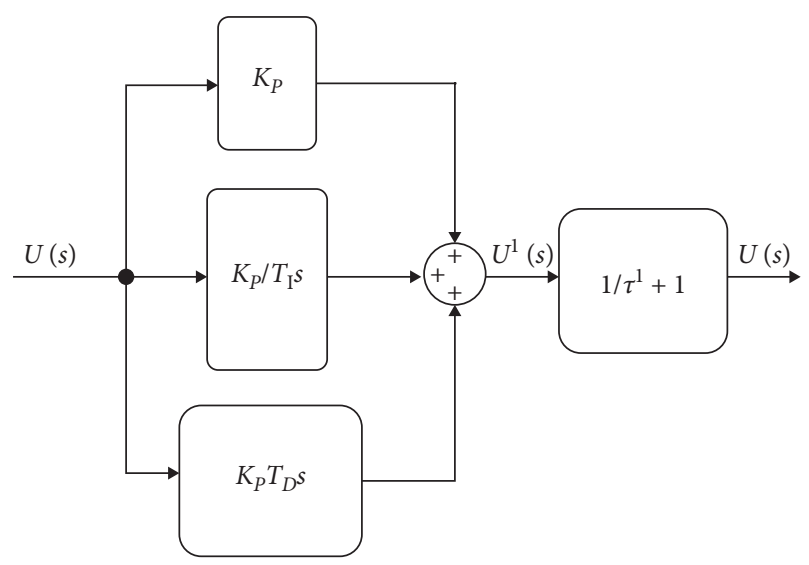

FIGURE 3: Differential passivation treatment.

Therefore, the discrete transfer function after passivation could be obtained:

$$
\frac{U(z)}{E(z)}=\frac{T}{T+\tau^{1}} \frac{m_{0}+m_{1} z^{-1}+m_{2} z^{-2}}{\left(1-z^{-1}\right)\left(1-\alpha z^{-1}\right)} .
$$

\section{Mathematical Model of the Injection System of the Injection Molding Machine}

Ajmeri and Ali [11] reviewed the controllers of injection molding machine. Although the intelligent control strategy is well-researched, its stability is the most critical aspect for an actual production machine. For example, in order to ensure the stability of production, the injection speed of an injection molding machine is mainly controlled using the conventional PID control. In order to verify the effectiveness of the improved PID controller to improve the control effect of the injection speed of the injection molding machine, the injection system of the machine with complex mathematical model and production conditions and a tendency to produce overshoot was selected as the control object, based on one of the world's best-selling Haitian Tianlong IAIIS series injection molding machines.

Figure 4 is the schematic diagram of the injection system of the Tianlong IAIIS series injection molding machine, including the hydraulic motor, servo valve, rotary encoder, flow sensor, reversing valve, and other important components. The open size and the open direction of the servo valve spool determined the size and the direction of the liquid flow in the hydraulic system, while the size and the direction of the liquid flow determined the moving speed and the moving direction of the injection screw, both of which determined the accuracy of the injection speed. Therefore, the position of the servo valve spool was taken as the variable of injection speed. The rotary encoder could obtain the speed of the servo motor in real time, and the flow sensor could obtain the flow passing through the valve in real time, both of which were used as the feedback values of the control system.

4.1. Mathematical Model for the Servo Valve. The servo valve power transmission system consisted of a servo motor and an internal gear. It was a second-order system with the current as the input value and the valve flow that was collected using the flow sensor as the output value. The transfer function could be obtained:

$$
D_{v}=\frac{Q_{0}}{I(s)}=\frac{K_{v}}{\left(\left(s^{2} / \omega_{v}^{2}\right)+\left(2 \varsigma_{v} / \omega_{v}\right) s+1\right)},
$$

where $K_{v}=Q_{n} \sqrt{P_{s} / P_{s n}} / I_{s n}$ is the flow gain, $P_{s}$ denotes the actual oil pressure value, $P_{s n}$ represents the rated pressure drop, $Q_{0}$ is the no-load flow, $Q_{n}$ is the rated flow, $\omega_{v}$ represents the valve angle frequency, $I_{s n}$ is the rated current, and $\varsigma_{v}$ is the damping coefficient.

4.2. Mathematical Model of the Injection Cylinder. The injection cylinder was hydraulically driven. Its working principle is shown in Figure 5. According to Newton's Second Law of Motion, Force, and Acceleration, ignoring other nonlinear effects, the differential equation of motion of the injection cylinder could be obtained:

$$
\mathrm{AP}=m \frac{\mathrm{d} v}{\mathrm{~d} t}+\mathrm{Bv}+F_{l}
$$

where $F_{l}$ is the external load, $v$ is the speed of the injection piston, $A$ is the effective area of pressure, $P$ is the internal pressure driving the movement of the hydraulic cylinder, and $B$ is the damping coefficient.

The injected liquid $Q$ drove the injection rod to move by changing the volume on both sides of the cylinder. According to the conservation of the total liquid flow, the differential equation could be obtained:

$$
Q=A v+C_{l} P+\frac{V_{a}}{\beta} \frac{\mathrm{d} P}{\mathrm{~d} t},
$$




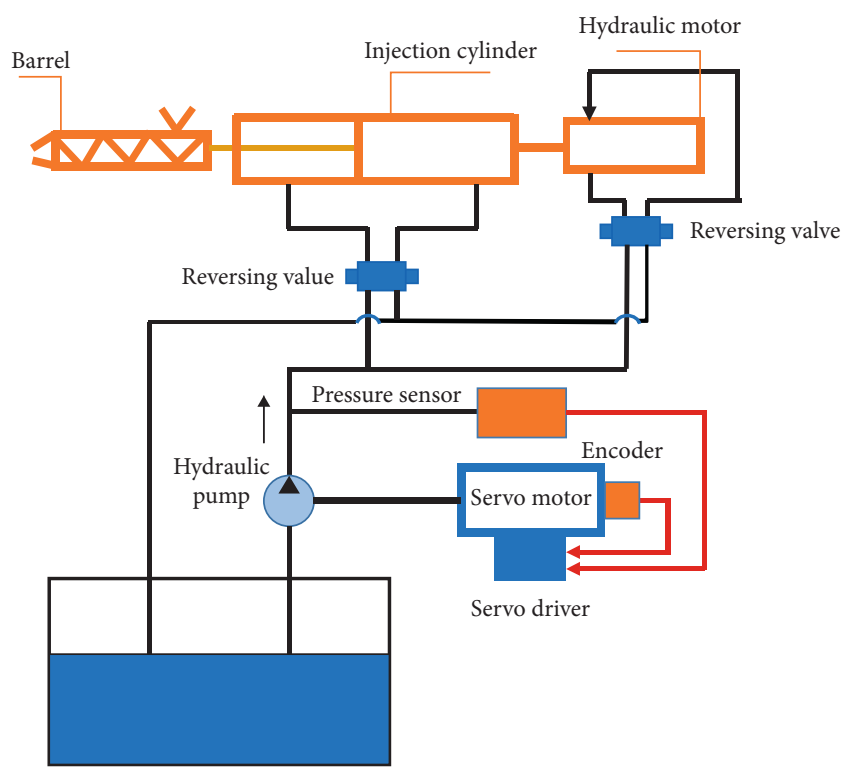

FIGURE 4: Injection system schematic of Tianlong IAIIS series injection molding machine.

where $C_{l}$ represents the sealing coefficient, $\beta$ denotes the equivalent volume of the liquid, $V_{a}$ represents the actual

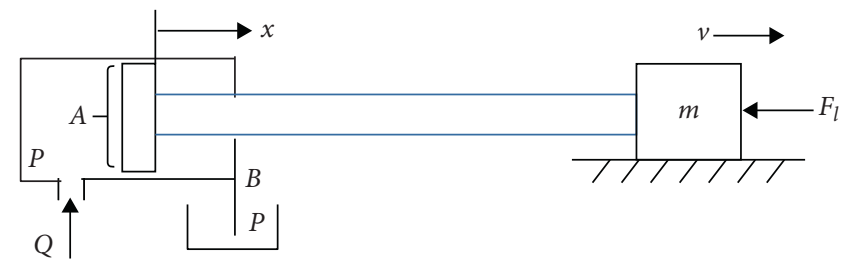

FIgURE 5: Schematic of hydraulic cylinder.

volume of the liquid, $Q$ is the total flow in the cylinder, and $\left(V_{c} / \beta\right)(\mathrm{d} P / \mathrm{d} t)$ is the volume change rate of the liquid in the cylinder when the screw was moving.

After the Laplace transformation of (9) and (10), the function in the complex field could be obtained:

$$
\begin{aligned}
\mathrm{AP}(s) & =(\mathrm{ms}+B) V(s)+F_{l}(s), \\
\mathrm{Q}(s) & =\mathrm{AV}(s)+\left(C_{l}+s \frac{V_{a}}{\beta}\right) P(s) .
\end{aligned}
$$

After substituting (11) into (12), the following equation could be obtained:

$$
Q(s)=\frac{F_{l}(s)}{A}\left(C_{l}+\frac{V_{a}}{\beta} s\right)+\frac{V(s)}{A}\left[\frac{V_{a}}{\beta} \mathrm{Ms}^{2}+\left(\frac{V_{a}}{\beta}+C_{l} M\right) s+\left(A^{2}+C_{l} B\right)\right] .
$$

If load $F_{l}$ was 0 , the transfer function of the hydraulic cylinder was

$$
G(s)=\frac{V(s)}{Q(s)}=\frac{A}{A^{2}+C_{l} B} \cdot \frac{1}{\left(s / w_{n}\right)^{2}+\left(2 \varsigma_{c} / w_{n}\right) s+1},
$$

where the natural frequency is $\omega_{n}=\sqrt{\left(A^{2}+C_{l} B\right) / \mathrm{mV}_{a}}$, and the damping ratio is $\varsigma_{n}=\left(\omega_{n} / 2 \beta\right) \cdot\left(V_{c} B+m \beta C_{l} / A^{2}+C_{l} B\right)$.
4.3. Transfer Function of the Injection System. According to the transfer functions of the servo valve and the injection cylinder, the transfer function of the injection speed could be obtained:

$$
G_{v}(s)=\frac{Q(s)}{I(s)} \cdot \frac{V(s)}{Q(s)}=\frac{\mathrm{AK}_{v}}{A^{2}+C_{l} B} \cdot \frac{1}{\left[\left(s / w_{n}\right)^{2}+\left(2 \varsigma_{c} / w_{n}\right) s+1\right] \cdot\left[\left(s / w_{v}\right)^{2}+\left(2 \varsigma_{v} / w_{v}\right) s+1\right]} .
$$

The identification of the key parameters in the transfer function faced cumbersome and complicated problems with different parameters due to the nonlinear characteristics of the input and the output. For the above problems, the Taylor Formula was used for analysis, and the ideal state assumptions were as follows. (1) The servo motor was a firstorder link, (2) during the injection process, liquid leakage was negligible, and (3) the working range of the servo valve spool was narrow and controllable.

When identifying the mathematical model of an injection system, different methods are often used to identify the servo valve model, such as the pulse response method and the parameter optimization method. The mathematical model constructed in this study was highly consistent with the fourth-order linear model for the injection speed of the injection molding machine established by Wang and Shen [14]. Hence, the mathematical model used in the simulation described below was

$$
G_{v}(s)=\frac{2.167 \times 10^{11}}{(s+130)(s+1140)\left[(s+390)^{2}+1138^{2}\right]} .
$$




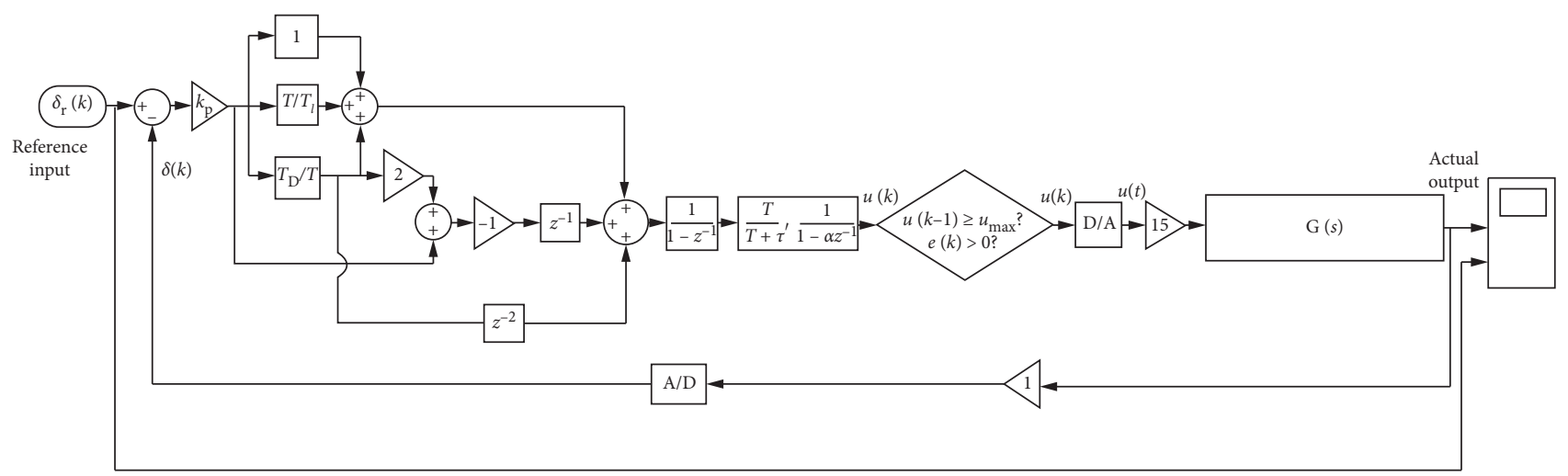

FIGURE 6: Simulation of improved PID control.

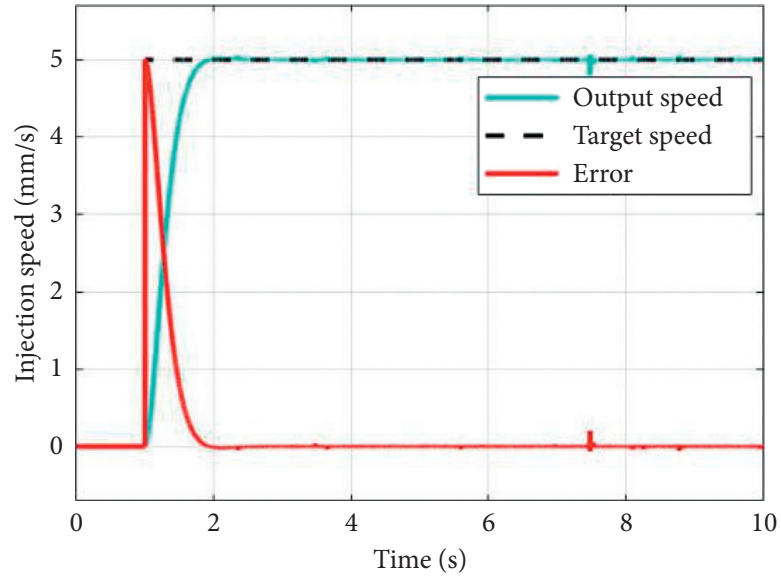

(a)

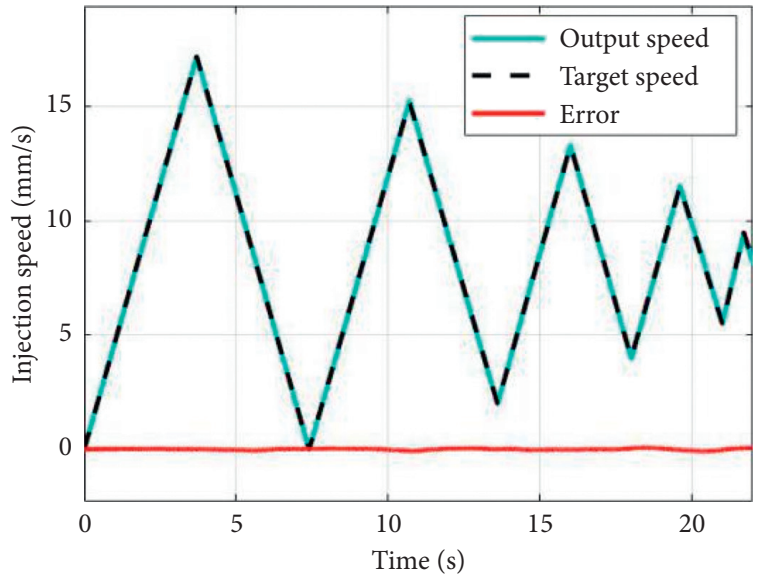

(b)

Figure 7: Improved PID simulation results. (a) Step response simulation. (b) Composite response simulation.

\section{Simulation}

The mathematical model of the injection speed was constructed as described above, and it was combined with the improved integral term and the differential term. The diagram of the injection speed in Simulink using improved PID control is shown in Figure 6.

In order to verify the control effect of the improved PID controller, a target speed of $5 \mathrm{~mm} / \mathrm{s}$ was input to the controller, and the injection speed rise time was $0.5 \mathrm{~s}$, as shown in Figure $7(\mathrm{a})$. The system steady-state time was $1.1 \mathrm{~s}$. The speed response had no overshoot and the steady-state error was 0. As shown in Figure 7(b), the maximum value of the output speed was $17 \mathrm{~mm} / \mathrm{s}$. The combined signal gradually decreased, and the error of the injection speed was almost zero. The simulation showed that the improved PID controller not only had a higher response speed to the injection speed, but also effectively reduced errors.

\section{Experimental Verification}

The performance of the improved PID controller was verified using experiments, the experiments are conducted in IA1200, Haitian Co., Ltd., as shown in Figure 8. The working

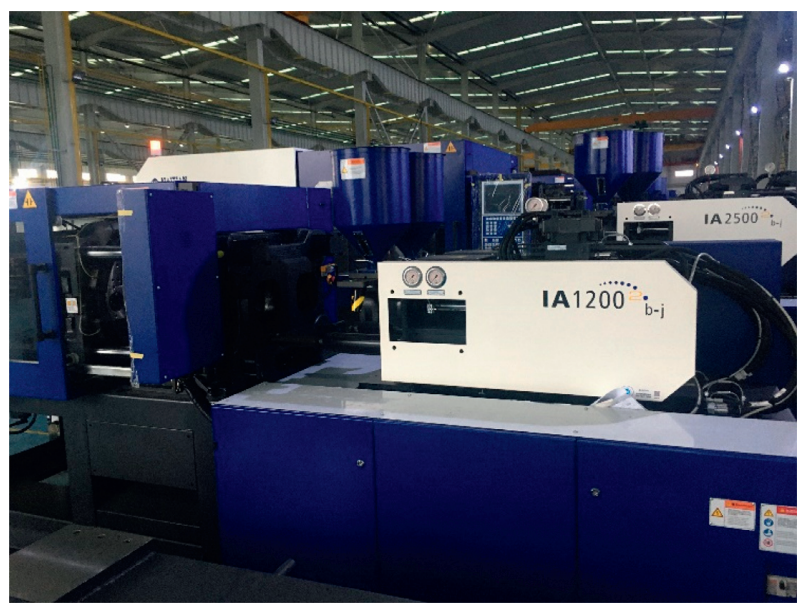

FIGURE 8: IA1200 series.injection molding machine.

process was as follows. The difference between the actual output speed and the desired speed was processed using the improved PID control strategy, the results of which were then used as the control signal. The digital signal was converted into an analog signal, which was taken as the input 


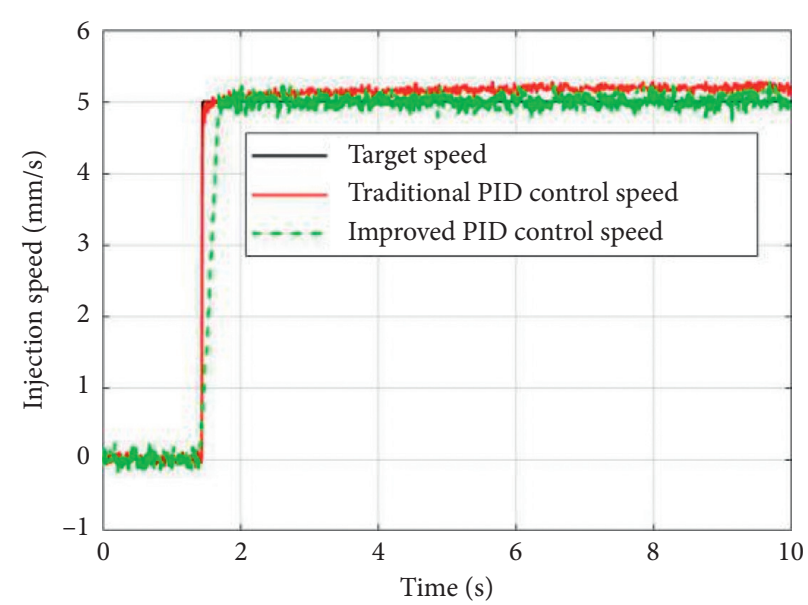

(a)

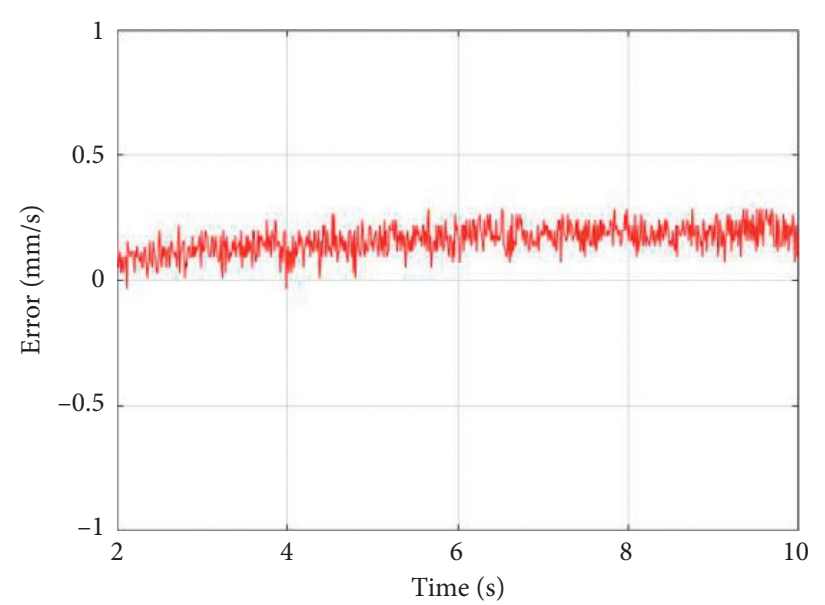

(b)

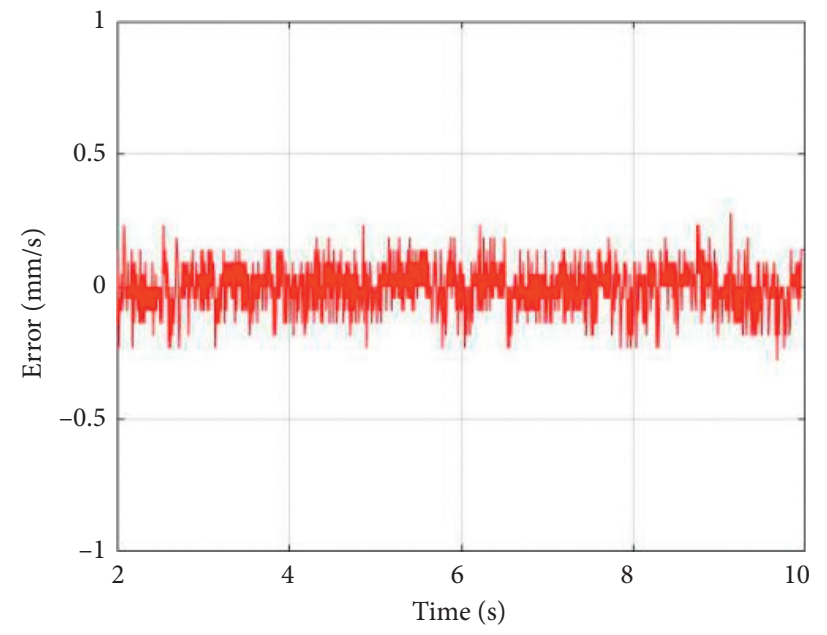

(c)

Figure 9: Step response test. (a) Comparison of three speed curves. (b) The error of traditional PID control. (c) The error of improved PID control.

voltage of the motor. The injection speed curve of the injection port was detected using a flow sensor. Next, the $A / D$ converter converted the continuous signal into discrete signals and sent it to the controller. The obtained signal was compared with the desired value, and the difference between the two was used as the error value $[11,12]$. In order to make the experiment closer to the actual production, the step signal, which is the most commonly used signal in actual production, is selected as the input signal. A complex arbitrary amplitude signal is used as the input signal to further verify the control effect of the improved PID controller.

6.1. Step Signal Test. Figure 9(a) shows the expected speed curve of the injection system of the injection molding machine with a duration of $10 \mathrm{~s}$ and a steady-state value of $5 \mathrm{~mm} / \mathrm{s}$, the results of traditional PID control and improved PID control. In traditional PID control, the response time of injection speed is $0.02 \mathrm{~s}$. Compared with the expected speed curve, the steady-state error center line is about $0.2 \mathrm{~mm} / \mathrm{s}$, and the injection speed curve has an upward trend with the increase of time (i.e., the larger error), as shown in Figure 9(b). In the improved PID control, the injection speed response time is $0.2 \mathrm{~s}$. Compared with the expected speed curve, the steady-state error center line is almost 0 , and the control error does not increase with time, as shown in Figure 9(c). In actual production, the steady-state error and stability of the injection speed are more important parameters than the response time. Therefore, the improved PID control strategy can significantly improve the injection accuracy of the injection speed.

6.2. Signal Test with Arbitrary Amplitude. Figure 10(a) shows the arbitrary injection speed curve (duration $18 \mathrm{~s}$, speed range $0 \sim 16.5 \mathrm{~mm} / \mathrm{s}$ ), which is composed of sine signal, ramp signal, constant signal, and so on, result of the traditional PID control and the improving PID control. As shown in Figure 10(b), the actual output injection speed curve of traditional PID control is compared with the expected speed curve, and the error center line range is $-1.72 \sim 0.75 \mathrm{~mm} / \mathrm{s}$, and the followability is poor, which 


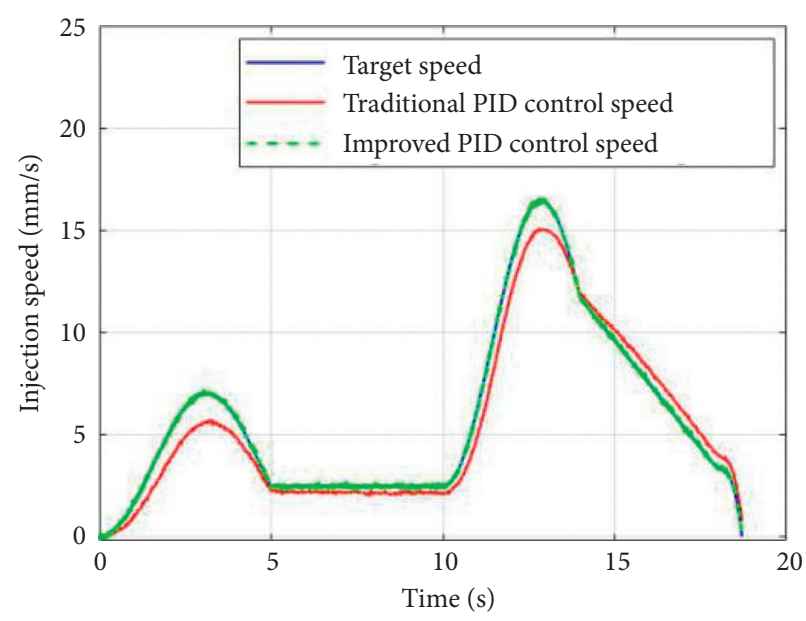

(a)

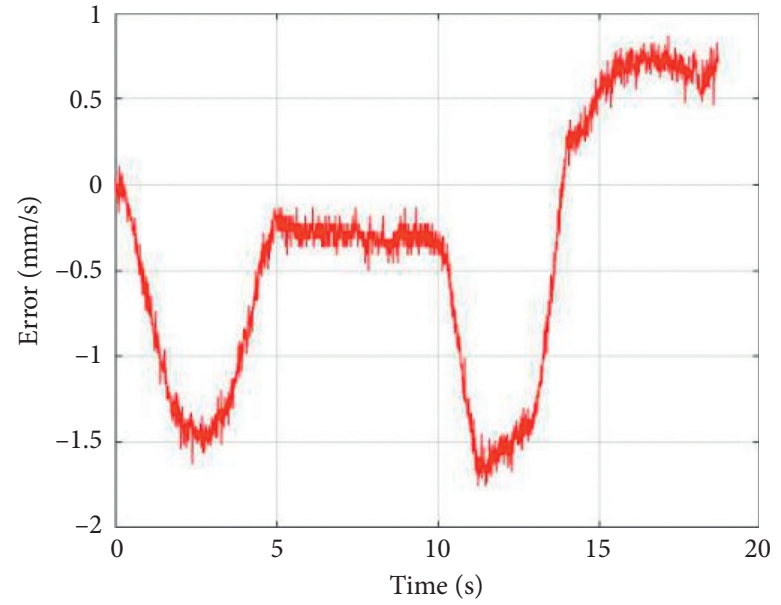

(b)

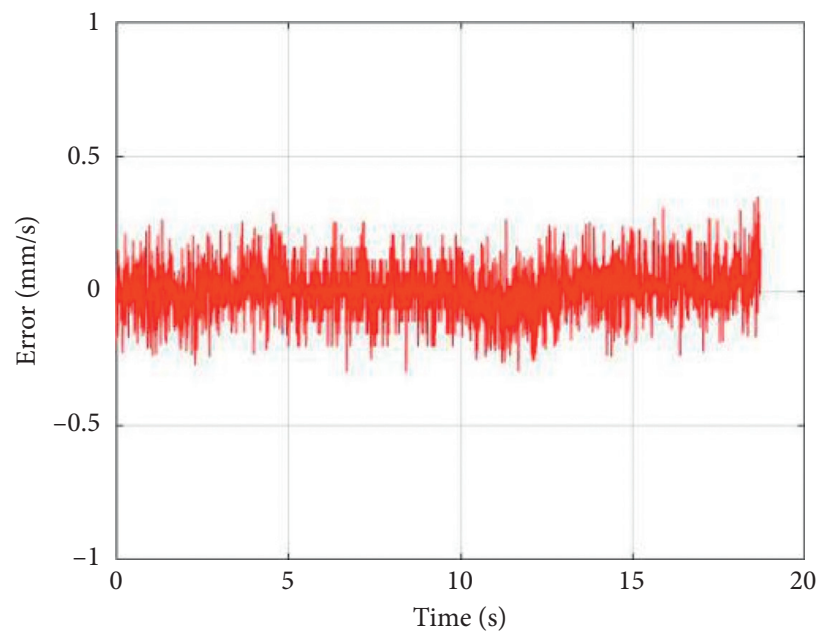

(c)

Figure 10: Random wave response test. (a) Comparison of three speed curves. (b) The error of traditional PID control. (c) The error of improved PID control.

cannot meet the requirement of the more complicated injection speed. As shown in Figure 10(c), when the improved PID controller is performed, the steady-state error center line is almost $0 \mathrm{~mm} / \mathrm{s}$. The improved PID controller also has better followability. It can be observed that the improved PID controller can track the injection speed well and the error is almost zero for any desired curve of amplitude.

\section{Conclusions}

First, the shortcomings of the conventional integral and differential terms of PID control were analyzed and the unsaturated integral algorithm was used to replace the integral term in the conventional PID algorithm. Then, the differential term was passivated by adding a low-pass filter in the conventional PID controller in order to improve the conventional PID. Next, the improved PID was used to improve the control accuracy of the injection speed of the injection molding machine, and Matlab/ Simulink was used to simulate and analyze the control effect. The simulation results showed that the injection speed rise time was $0.5 \mathrm{~s}$ and the system steady-state time was $1.1 \mathrm{~s}$. The speed response had no overshoot and the steady-state error was 0. Finally, the control effect was verified using experiments, the results of which showed that for the $5 \mathrm{~mm} / \mathrm{s}$ step target speed curve, the injection speed response time was $0.2 \mathrm{~s}$ without overshoot and the steady-state error midline was almost reduced to $0 \mathrm{~mm} / \mathrm{s}$ from $0.66 \mathrm{~mm} / \mathrm{s}$ for the PID control before improvement. For the target speed curve with an arbitrary amplitude, the error midline of the injection speed was reduced to almost 0 from the range of $-1.72 \mathrm{~mm} / \mathrm{s}$ to $0.75 \mathrm{~mm} / \mathrm{s}$ for the PID control before improvement. Therefore, with the improved PID controller, the injection speed of the injection molding machine could rapidly and accurately track the desired speed curve.

\section{Data Availability}

The raw/processed data required to reproduce these findings cannot be shared at this time as the data also form part of an ongoing study. 


\section{Conflicts of Interest}

The authors declare that they have no conflicts of interest.

\section{Acknowledgments}

The authors would like to acknowledge the financial support of the Zhejiang Provincial Natural Science Foundation of China (Grant no. LGG21E050005) and the Key Research and Development Plan of Zhejiang Province (Grant no. 2020C01113).

\section{References}

[1] D. Habineza, M. Rakotondrabe, and Y. Le Gorrec, "Bouc-wen modeling and feedforward control of multivariable hysteresis in piezoelectric systems: application to a 3-DoF piezotube scanner," IEEE Transactions on Control Systems Technology, vol. 23, no. 5, pp. 1797-1806, 2015.

[2] H. Chien, Y. J. Wen, and R. J. Yeau, "Design and control of a long-traveling nano-positioning stage," Precision Engineering, vol. 34, no. 3, pp. 497-506, 2010.

[3] H. Tang and Y. Li, "Feedforward nonlinear PID control of a novel micromanipulator using Preisach hysteresis compensator," Robotics and Computer-Integrated Manufacturing, vol. 34, pp. 124-132, 2015.

[4] F.-C. Wang, Y.-C. Tsai, C.-H. Hsieh, L.-S. Chen, and C.-H. Yu, "Robust control of a two-Axis piezoelectric nano-positioning stage," IFAC Proceedings Volumes, vol. 44, no. 1 , pp. 3539-3544, 2011.

[5] W. Zhu, F. Yang, and X. Rui, "Robust independent modal space control of a coupled nano-positioning piezo-stage," Mechanical Systems and Signal Processing, vol. 106, pp. 466478, 2018.

[6] C. Leon, S. Bijan, B. Umesh et al., "Development and control of a two DOF linear-angular precision positioning stage," Mechatronics, vol. 32, pp. 34-43, 2015.

[7] C. Y. Xie, "A neural networks based model for rate-dependent hysteresis for piezoceramic actuators," Sensors and Actuators A: Physical, vol. 143, 2008.

[8] L. Li, C.-X. Li, G. Gu, and L.-M. Zhu, "Positive acceleration, velocity and position feedback based damping control approach for piezo-actuated nanopositioning stages," Mechatronics, vol. 47, pp. 97-104, 2017.

[9] K. Cai, Y. Tian, F. Wang, D. Zhang, and B. Shirinzadeh, "Development of a piezo-driven 3-DOF stage with T-shape flexible hinge mechanism," Robotics and Computer-Integrated Manufacturing, vol. 37, no. 3, pp. 125$138,2016$.

[10] M. Han, H. Wu, Y. Song, Y. Cheng, and H. Handroos, "Intelligent method for tuning H2 PID controller of water hydraulic manipulator of CFETR blanket maintenances," Industrial Robot: An International Journal, vol. 43, no. 2, pp. 164-171, 2016.

[11] M. Ajmeri and A. Ali, "Direct synthesis based tuning of the parallel control structure for integrating processes," International Journal of Systems Science, vol. 46, no. 13, pp. 2461-2473, 2015.

[12] R. E. Nunn and C. P. Grolman, "Adaptive process control for injection molding," Journal of Reinforced Plastics \&Composites, vol. 9, no. 3, pp. 282-298, 1990.

[13] M. R. Kamal, A. E. Varela, and W. I. Patterson, "Control of part weight in injection molding of amorphous thermoplastics," Polymer Engineering \& Science, vol. 39, no. 5, pp. 940-952, 1999.

[14] K. K. Wang and S. F. Shen, "Injection molding projection progress report," Cornel University, Ithaca, NY, USA, 1985. 